\title{
Impacto de las microfinanzas en el crecimiento de las mipymes (alojamientos, hostales y hoteles) del Callejón de Huaylas, departamento de Ancash (2010-2014)
}

Impact of microfinance on growth of msmes (accommodation, hostels and hotels) Callejón de Huaylas, Ancash department (2010-2014)

Cerafín Primitivo Toledo Cena ${ }^{1}$

\section{RESUMEN}

El objetivo central del estudio es conocer la incidencia de las micro finanzas en el crecimiento de las MIPYMES (Alojamientos, hostales y hoteles) del Callejón de Huaylas, departamento de Ancash (2010-2014). El diseño metodológico corresponde a una investigación no experimental basada en una aplicación censal, que ha permitido establecer el grado de relación entre las variables de estudio. Los resultados obtenidos confirman que, el 38.4\% de las MIPYMES tienen acceso al crédito y consiguientemente renuevan sus bienes de capital; un 50.3\% incrementó su valor, sin embargo, el retorno de su capital es lento; un $57.6 \%$ crecieron permitiéndoles cumplir su hoja de dudas, no obstante el ROA es variable; y, que la colocación de capitales frescos favorece la creación de empleos decentes. Finalmente, se concluye que, las MIPYMES estudiadas relativamente crecen poco, siendo la rigidez de la cartera de riesgos un factor determinante para el acceso a los créditos; el lento retorno de la riqueza para los propietarios, que limita la disponibilidad de efectivo; el rendimiento lento de los activos, como tercer factor, que limita la disponibilidad del efectivo y finalmente que la inyección de capitales a las MIPYMES favorece la generación de empleos en la zona.

Palabras clave: microfinanzas; crecimiento; MIPYMES.

\section{ABSTRACT}

The main objective of the study is to know the incidence of micro finance in the growth of MIPYMES (Lodging, hostels and hotels) of Callejón de Huaylas, department of Ancash (2010-2014). The methodological design corresponds to a non-experimental research based on a census application, which has allowed establishing the degree of

1 Universidad Nacional Santiago Antúnez de Mayolo. Huaraz, Perú. 
relationship between the study variables. The results confirm that $38.4 \%$ of MIPYMES have access to credit and consequently renew their capital assets; 50.3\% increased its value, however, the return of its capital is slow; $57.6 \%$ grew allowing them to fulfill their doubts sheet, however the ROA is variable; And that the placement of fresh capital favors the creation of decent jobs. Finally, it is concluded that the MSYMES studied relatively grow little, being the rigidity of the risk portfolio a determining factor for access to credits; The slow return of wealth to owners, which limits the availability of cash; The slow performance of assets, as a third factor, which limits the availability of cash and finally that the injection of capital to MIPYMES favors the generation of jobs in the area.

Keywords: microfinance; growth; MIPYMES.

\section{ICHIKLLACHAW}

Kay musyay ashipakuyqa imanawmi mikru hwinansas MIPYMESpa wiñaynintam riqitsikamun (tukuy puñuna wayikuna) Waylas Raqrachaw, Anqash (2010-2014) kanqantam. Kay musyapakuyqa mana ikpirimintal nishqanwanmi rurakashqa, tsaypaqqa tapuytam wanakashqa, tsaywanmi imanaw tinkuyninta hurqaqashqa. Kay yarquynin 38.4\% MIPYMES nishqan mañakuyninmanmi awqan, tsaymi kaqninkunata yapay mushuqyaatsiyan; 50.3\%kama tsanintsaynin witsashqa, tsaynaw kaykar wachay qillayninqa nakanmi; 57.6\%tum wiñayashqa tsaywanran tsuyantsaarin, tsaynaw kaykar ROA nishqanpis tumarinran, qipa qillay wachay churayninqa yatsatsikuqkunapa uryaynintam yuritsin. Uchukllachaw nishqaqa rikaatsimantsik imanawmi MIPYMES nishqan ichikllapayan wiñan, mana qillay kanqanmi allitsuqa, tsayraykurmi mana allitsu imallatapis hurqakunapaq, tsaymi kikin kapuqkunaman nakaypa kutin, hinamanpis MIPYMESman kapital yaykuynin uryakuna yuriynintam alliyaatsi.

Pushaq shimikuna: mikru hwinansas nishqan; MIPYMESpa wiñaynin.

\section{INTRODUCCIÓN}

El estudio tiene como propósito determinar la incidencia de las microfinanzas en el crecimiento de las MIPYMES (alojamientos, hostales y hoteles) del Callejón de Huaylas, departamento de Ancash, durante los períodos 2010-2014. Actualmente, el problema de estudio se mantiene latente, es decir, el servicio de alojamiento, hoteles y hostales, no tiene acceso al crédito financiero por las altas tasas de interés, el riesgo de incumplimiento de la hoja de deudas y las trabas de respaldo que exigen las microfinanzas, entre otros factores.

Las MIPYMES en el Perú (Espinoza. 2010), enfrentan tres grandes problemas: El primero es la inaccesibilidad a los mercados nacionales e internacionales. El segundo gran problema de las PYMES en el Perú -y vinculado al primero- es que los pequeños 
y microempresarios no tienen dinero en la mano para invertir cada vez más y poder acceder a los mercados nacionales e internacionales; es decir, estamos frente al problema de las microfinanzas. Un tercer problema de las PYMES en el Perú -e igualmente vinculado al primero-, es la casi absoluta ausencia de la ciencia, tecnología e innovación en la vida cotidiana de la empresa. En el contexto anterior, las MIPYMES ubicadas en el Callejón de Huaylas, presentan una caracterización muy similar a las del resto del país, esto es que, se enfrentan a una cartera de créditos muy rígida, el retorno del capital y el rendimiento de los activos fijos es muy lento y, consiguientemente, se dispone de escasos activos líquidos.

Los objetivos específicos del estudio son: Establecer la magnitud de colocaciones de capitales por parte de los agentes financieros, el incremento de la riqueza de los propietarios, la reposición del capital de trabajo y la generación de empleos en las MIPYMES (alojamientos, hoteles y hostales) en el Callejón de Huaylas en el período 2010-2014.

La investigación se justifica porque nos permite:

Planificar la permanencia de las MIPYMES en el Callejón de Huaylas, a fin de obtener indicadores que nos permitan proyectar su crecimiento, sobre tendencias sólidas y sostenibles. Atraer nuevas y frescas inversiones en actividades económicas propias de las MIPYMES en el Callejón de Huaylas, y consiguientemente, alentar y promover la generación de oportunidades laborales decentes; diversificar la naturaleza operativa de las MIPYMES en el Callejón de Huaylas, orientando los flujos de caja libre a sectores de producción innovados o genuinos.

Por lo tanto, el estudio abarca aspectos relevantes relacionados a la tasa de crecimiento de las MIPYMES (alojamientos, hostales y hoteles) del Callejón de Huaylas, el nivel de reposición del capital de trabajo, el incremento de bienes de capital, la disponibilidad de los flujos de efectivo y la generación de empleos decentes.

\section{MATERIALES Y MÉTODOS}

La investigación es de diseño no experimental y se desarrolla sobre la base de una muestra. Así mismo, aplica los parámetros financieros para comparar los indicadores obtenidos con aquellos que se muestran en los estados financieros de las MIPYMES, y se deduce su impacto. La investigación tiene un nivel correlacional, y permite establecer el grado de relación entre las variables independientes con la variable dependiente.

La población de la investigación está conformada por 247 MIPYMES, que actualmente vienen operando en el Callejón de Huaylas. Para este efecto se ha considerado las provincias de Huaraz, Recuay, Carhuaz, Yungay y Huaylas. El método para definir la muestra (151), está basado en el muestreo probabilístico; para ello se ha definido los elementos de la población en estudio, siendo el elemento base de análisis el administrador o gerente de cada MIPYME. 
Los datos recopilados se procesan al SPSS-23 para los reportes y corrida de modelos con las pruebas estadísticas correspondientes, para luego contrastarlas y determinar las conclusiones y recomendaciones objeto de la investigación.

Los métodos considerados en el estudio son:

Método Inductivo: Permite indagar y evaluar las implicancias financieras en relación a la optimización de las microfinanzas en las MIPYMES del Callejón de Huaylas.

Método Deductivo: Contribuye a la determinación de los indicadores.

Método Analítico: Se utiliza parámetros financieros para desmenuzar la deducción y conclusión del fenómeno en estudio.

Para ello se aplica la técnica de la encuesta a 151 MIPYMES y su instrumento es el cuestionario, y, el análisis documental (30 Estados Financieros) y su instrumento es el análisis financiero.

La investigación ha observado rigurosamente los aspectos éticos de su realización y obtenido los consentimientos de derechos de autoría de las referencias consultadas.

\section{RESULTADOS}

Tabla 1. Acceso al crédito y reposición de bienes de capital en las MIPYMES 2010-2014

\begin{tabular}{|c|c|c|c|c|c|c|}
\hline \multirow{3}{*}{$\begin{array}{l}\text { En caso de haber realizado la re- } \\
\text { posición o adquisición de nuevos } \\
\text { bienes de capital ¿Cuál sería el fac- } \\
\text { tor determinante para realizar esta } \\
\text { acción? }\end{array}$} & \multicolumn{6}{|c|}{$\begin{array}{c}\text { ¿En los últimos cinco años su negocio } \\
\text { accedió al crédito de las microfinancieras } \\
\text { del medio? }\end{array}$} \\
\hline & \multicolumn{2}{|c|}{ Sí } & \multicolumn{2}{|c|}{ No } & \multicolumn{2}{|c|}{ Total } \\
\hline & $\mathrm{n}$ & $\%$ & $\mathrm{n}$ & $\%$ & $\mathrm{n}$ & $\%$ \\
\hline $\begin{array}{l}\text { Se adquieren en proporción a las } \\
\text { ventas }\end{array}$ & 28 & 25.0 & 30 & 26.8 & 58 & 51.8 \\
\hline $\begin{array}{l}\text { Se adquieren en la proporción de la } \\
\text { tasa de crecimiento económico }\end{array}$ & 43 & 38.4 & 11 & 9.8 & 54 & 48.2 \\
\hline Total & 71 & 63.4 & 41 & 36.6 & 112 & 100.0 \\
\hline
\end{tabular}


De la tabla 1, se puede observar que el 38.4\% de los empresarios tuvieron acceso al crédito de las microfinancieras y adquirieron bienes de capital según la tasa de crecimiento económico; además se puede observar que el 26.8\% no tiene acceso al crédito de las microfinancieras y adquiere bienes de capital en proporción a sus ventas. De lo observado en el gráfico se concluye que la rigidez de la cartera de riesgos limita el requerimiento de créditos para nuevos bienes de capital. Además, realizando la prueba estadística de chi cuadrado a un nivel de confianza de $95 \%$, se observa que existe una relación significativa entre las variables de estudio, pues el P-valor es menor al 5\%.

Tabla 2. Valor de la MIPYME y retorno del capital 2010-2014

\begin{tabular}{|c|c|c|c|c|c|c|c|c|}
\hline \multirow{3}{*}{$\begin{array}{c}\text { ¿En los últimos } \\
\text { cinco años se } \\
\text { incrementó } \\
\text { el valor de la } \\
\text { empresa que } \\
\text { administra? }\end{array}$} & \multicolumn{8}{|c|}{$\begin{array}{l}\text { En los últimos cinco años ¿Cuál ha sido el comporta- } \\
\text { miento del retorno sobre su capital invertido ROE)? }\end{array}$} \\
\hline & \multicolumn{2}{|c|}{ En aumento } & \multicolumn{2}{|c|}{ Variable } & \multicolumn{2}{|c|}{ En descenso } & \multicolumn{2}{|c|}{ Total } \\
\hline & $\mathrm{n}$ & $\%$ & $\mathrm{n}$ & $\%$ & $\mathrm{n}$ & $\%$ & $\mathrm{n}$ & $\%$ \\
\hline Sí & 41 & 27.2 & 76 & 50.3 & 20 & 13.2 & 137 & 90.7 \\
\hline No & 1 & 0.7 & 7 & 4.6 & 6 & 4.0 & 14 & 9.3 \\
\hline Total & 42 & 27.8 & 83 & 55.0 & 26 & 17.2 & 151 & 100.0 \\
\hline
\end{tabular}

En la tabla 2 se puede observar que el 50.3\% de los empresarios manifiestan que su empresa aumentó de valor, pero que también el ROE es variable. Además se puede observar que el 27.2\% manifiesta que aumentó de valor su empresa y también aumentó el ROE. De lo observado en el gráfico se concluye que el lento retorno del patrimonio deseable limita la disponibilidad de los flujos de efectivo. Además, realizando la prueba estadística de chi cuadrado a un nivel de confianza de $95 \%$, se observa que existe una relación significativa entre las variables de estudio, pues el P-valor es menor al 5\%. 
Tabla 3. Tasa de crecimiento y retorno de los activos en las MIPYMES 2010-2014

En relación a su tasa de cre-En los últimos cinco años: ¿cuál ha sido el comporcimiento registrado en los úl- tamiento del retorno sobre sus activos (ROA)? timos cinco años. ¿Considera que esto le permitió honrar su En aumenhoja de deudas con las micro- to Variable En descenso Total financieras $\sin$ registrar retra-

\begin{tabular}{lrrrrrrrrc} 
so alguno de alguna manera? & $\mathrm{n}$ & $\%$ & $\mathrm{n}$ & $\%$ & $\mathrm{n}$ & $\%$ & $\mathrm{n}$ & $\%$ \\
\hline Favorable & 32 & 21.2 & 87 & 57.6 & 5 & 3.3 & 124 & 82.1 \\
Desfavorable & 8 & 5.3 & 14 & 9.3 & 5 & 3.3 & 27 & 17.9 \\
Total & 40 & 26.5 & 101 & 66.9 & 10 & 6.6 & 151 & 100.0 \\
\hline & $\mathrm{X}^{2}=8.259$ & $\mathrm{gl}=2$ & \multicolumn{6}{c}{ P-valor=0.016 }
\end{tabular}

En la tabla 3, se puede observar que el 57.6\% de los empresarios manifiesta que la tasa de crecimiento de su empresa les ayudó a pagar sus deudas favorablemente, a pesar que el ROA es variable. Además se puede observar que el 21.2\% manifiesta que la tasa de crecimiento permitió pagar sus deudas y que el ROA está en aumento. De lo observado en el gráfico se concluye que el lento rendimiento sobre los activos deseables limita la reposición fluida del capital de trabajo; además realizando la prueba estadística de chi cuadrado a un nivel de confianza de $95 \%$, se observa que existe una relación significativa entre las variables de estudio, pues el $\mathrm{P}$-valor es menor al $5 \%$.

Tabla 4. Valor de la MIPYME y estabilidad laboral 2010-2014

\begin{tabular}{|c|c|c|c|c|c|c|}
\hline \multirow{3}{*}{$\begin{array}{l}\text { De su actual plani- } \\
\text { lla de trabajadores } \\
\text { ¿Cuántos son esta- } \\
\text { bles? }\end{array}$} & \multicolumn{6}{|c|}{$\begin{array}{c}\text { ¿En los últimos cinco años se incrementó el valor de la } \\
\text { empresa que administra? }\end{array}$} \\
\hline & \multicolumn{2}{|c|}{$\mathrm{Si}$} & \multicolumn{2}{|c|}{ No } & \multicolumn{2}{|c|}{ Total } \\
\hline & $\mathrm{n}$ & $\%$ & $\mathrm{n}$ & $\%$ & $\mathrm{n}$ & $\%$ \\
\hline Ninguno & 30 & 19.9 & 10 & 6.6 & 40 & 26.5 \\
\hline $50 \%$ & 57 & 37.7 & 3 & 2.0 & 60 & 39.7 \\
\hline $80 \%$ & 28 & 18.5 & 1 & 0.7 & 29 & 19.2 \\
\hline $100 \%$ & 22 & 14.6 & 0 & 0.0 & 22 & 14.6 \\
\hline Total & 137 & 90.7 & 14 & 9.3 & 151 & 100.0 \\
\hline
\end{tabular}


En la tabla 4, se puede observar que el $37.7 \%$ de los empresarios manifiestan que el valor de su empresa aumentó y que el 50\% de sus trabajadores están estables en planilla. Además se puede observar que el 70.9\% de los empresarios tienen a sus trabajadores estables y en planilla. De lo observado en el gráfico se concluye que la colocación de capitales incide favorablemente en la generación de empleos decentes; además, realizando la prueba estadística de chi cuadrado a un nivel de confianza de $95 \%$, se observa que existe una relación significativa entre las variables de estudio, pues el P-valor es menor al 5\%.

\section{DISCUSIÓN}

El modelo matemático diseñado para determinar la relación funcional de las variables intervinientes en la investigación, de acuerdo a la información proporcionada por los actuales administradores, gerentes o simplemente conductores de las MIPYMES (alojamientos, hostales y hoteles) del Callejón de Huaylas es: $\mathrm{Y}=\left(\mathrm{X}_{1} ; \mathrm{X}_{2} ; \mathrm{X}_{3} ; \mathrm{e}\right)$.

De las quince preguntas que se ha seleccionado para probar la validez de las hipótesis específicas y central, todas ellas confirman que los datos obtenidos no se distribuyen uniformemente; por lo tanto, las diferencias son estadísticamente significativas, dado que $\mathrm{p}<0.05$, entonces se rechaza la $\mathrm{H}_{0}$.

En este sentido, y como se puntualiza en la investigación, se desprende que entre las variables independientes $\mathrm{X}_{1} ; \mathrm{X}_{2} \mathrm{y}_{3}$ (Cartera de riesgos, retorno sobre el patrimonio y retorno sobre los activos) en relación a la variable dependiente: Crecimiento económico, existe una relación positiva relativamente baja. Este hecho estaría demostrando que las microfinanzas han incidido relativamente poco en el crecimiento económico de las MIPYMES (alojamientos, hostales y hoteles) en el Callejón de Huaylas en el período de estudio (2010-2014).

Este resultado corrobora las teorías: El factor determinante para la poca inyección de capitales por las microfinancieras $(\mathrm{MFs})$ es la denominada «Cartera de créditos contaminada», que está conformada por las deudas atrasadas o impagas; en este caso, si el repago registra un atraso de 30 días (Micro Rate y BID, 2003). En el sentido precedente, el estudio revela que un 32.7\% de las MIPYMES sostienen que las tasas de interés son elevadas y un $10.9 \%$ que se hallan en condiciones de morosidad. Por lo tanto, el estudio revela que una cartera rígida con tasas de interés elevadas no constituye un atractivo para promover la inversión en el sector hotelero del Callejón de Huaylas y se agrava con el cumplimiento oportuno de la hoja de deudas. Un retorno rápido y deseable sobre el capital o patrimonio invertido es una constante preocupación de cualquier 
empresario. Al no producirse ésta, es obvio que se genera un patrimonio que no está generando los flujos de caja necesarios que hagan posible un funcionamiento fluido de la empresa, en este caso, las MIPYMES (Micro Rate y BID, 2003). El estudio revela que el retorno del capital es muy lento y esta situación trae como consecuencia la escasez del efectivo. En este sentido, todo inversionista espera obtener el pronto retorno de su capital; esta limitación queda mostrada en los resultados del estudio, puesto que al ser lento el retorno del patrimonio, el valor de las MIPYMES (accionariado) también es lento o con altibajos. Un rendimiento eficiente y óptimo sobre los activos genera como resultado que la empresa pueda disponer de manera oportuna flujo de efectivos para ser destinados a la reposición oportuna y planificada del capital de trabajo, otorgándole a la empresa una mayor solidez financiera y económica frente a sus proveedores y acreedores(Micro Rate y BID, 2003). El estudio revela, así mismo que, el lento rendimiento de los activos no crea las condiciones apropiadas de funcionamiento por la falta de liquidez. El rendimiento eficiente de los activos genera el flujo de efectivo necesario para un funcionamiento operativo de calidad. Por lo tanto, los resultados obtenidos muestran que al no producirse un rendimiento óptimo de los activos, la limitada disposición de efectivo dificulta operativamente el normal funcionamiento de las MIPYMES estudiadas. El empleo decente (remuneración mínima, seguridad social y pensiones) constituye la base de toda sociedad civilizada que tiende al progreso y al desarrollo. El crecimiento económico de las empresas en este contexto se fortalece por los beneficios sociales, económicos y de jubileo para la masa de trabajadores (BCRP, Sucursal. Trujillo, 2013). El estudio revela que, un 26.5\% de los trabajadores de las MIPYMES estudiadas no tiene estabilidad laboral y solo un 14.6\% goza de estabilidad laboral. Esta realidad confirma nuestra hipótesis de estudio.

\section{CONCLUSIONES}

La incidencia de las microfinanzas en el crecimiento económico de las MIPYMES (alojamientos, hostales y hoteles) del Callejón de Huaylas durante el período 2010-2014, es relativamente poco, debido a la percepción de la rigidez de la cartera de créditos del sistema crediticio, las altas tasas de interés para inversiones y las penalidades que conlleva el incumplimiento de la hoja de deudas. Esta situación ha contraído las inversiones o la dinamización de las operaciones del sector servicio hostelero. No obstante ello, las MYPIMES registraron tasas de crecimiento positivas (94\%), siendo el rango de crecimiento de 1 al 5\% (44.4\% anual); del 6 al 10\% (38.7\%) anual; del 11 al 15\% (14.8\%) anual y más del 15\% crecieron $2.1 \%$ de las empresas consultadas; así mismo, 
el valor de la empresa se incrementó en un 90.7\%, hecho que les permitió afrontar sin dificultades su hoja de deudas con el sistema microfinanciero.

La investigación ha probado que las MYPIMES (alojamientos, hostales y hoteles) del Callejón de Huaylas accedieron al crédito financiero en un $63.6 \%$ y $36.4 \%$ no tuvieron acceso. Estos hechos estarían probando que la inyección de capitales para el servicio hostelero es relativamente débil, aun cuando el sondeo aplicado revela que las actuales administraciones de las MYPIMES en estudio y como consecuencia de su crecimiento variable han destinado sus utilidades (38.7\%) a la adquisición de nuevos bienes de capital (74.2\%); sin embargo, un significativo 76.8\% de los empresarios sostiene que los créditos obtenidos son insuficientes.

El retorno sobre el capital invertido es lento, debido a un comportamiento variable del precitado indicador, aun cuando su crecimiento económico aumentó en el período bajo estudio (2010-2014), solo un 38.8\% de los empresarios consultados afirma que se dispuso de los flujos de efectivo para sus operaciones de funcionamiento.

El ROA (rendimiento sobre sus activos) registra un comportamiento variable, no obstante ello el crecimiento de las MIPYMES los favoreció para cumplir satisfactoriamente con su hoja de deudas con el sistema microfinanciero, aun cuando 46.4\% de los empresarios consultados sostiene que financiaron sus proyectos y operaciones con sus recursos propios, aunados a la percepción de los mismos, en el sentido de que el financiamiento externo resulta de mayor rentabilidad para los propietarios.

Existe una relación de significación positiva entre la variable independiente generación de empleo y la variable dependiente crecimiento económico, esto es que, todo crecimiento económico de las MIPYMES (alojamiento, hostales y hoteles) del Callejón de Huaylas, favorece el incremento de empleos en condiciones decentes.

\section{REFERENCIAS BIBLIOGRÁFICAS}

Banco Central de Reserva del Perú. Sucursal Trujillo. 2013. Sintesis económicos Ancash. Departamento de estudios económicos.

Espinoza, Nemesio. 2010. Las pequeñas y microempresas como alternativas de solución al problema del desempleo y de la pobreza en el Perú. Lima: Universidad Nacional Mayor de San Marcos.

INEI. 2014. Estadísticas de la Micro, Pequeña y Mediana empresa. Lima. 
Micro Rate. Banco Interamericano de Desarrollo. 2013. Departamento de desarrollo sostenible. División de micro, pequeña y mediana empresa. Indicadores de desempeño para instituciones microfinancieras. Guía técnica. $3^{\circ}$ edición. Washington D.C

Fecha de recepción: 10 de julio de 2016

Fecha de aceptación: 20 de noviembre de 2016

\section{Correspondencia}

Cerafín Primitivo Toledo Cena

E-mail: peru9207serita@gmail.com 